\title{
Corrigendum
}

\section{Corrigendum to: Impact of reference change value (RCV) based autoverification on turnaround time and physician satisfaction}

Esther Fernández-Grande', Carolina Varela-Rodriguez², Luis Sáenz-Mateos³, Amparo Sastre-Gómez¹, Pilar García-Chico1, Teodoro J. Palomino-Muñoz ${ }^{* 1}$

'Servicio de Análisis Clínicos, Hospital General Universitario de Ciudad Real, Ciudad Real, Spain.

2Servicio de Medicina Preventiva, Hospital General Universitario de Ciudad Real, Ciudad Real, Spain.

${ }^{3}$ Servicio de Análisis Clínicos, Complejo Hospitalario de Navarra, Pamplona, Spain

*Corresponding author: tjavierpm@sescam.jccm.es

This is a correction for Biochemia Medica 2017;27(2): 342-49. DOI: https://doi.org/10.11613/BM.2017.037 .

Since the publication of this article, the authors have noticed that the surname of the second au- thor in the byline was published incorrectly. The correct byline is presented above. The authors apologize for any inconvenience caused to the readers. 